\title{
Pseudoaneurisma da fibrosa intervalvar mitro-aórtica: um estudo de caso
}

\author{
Pseudoaneurysm of the mitro-aortic intervalve fibrosa: a case study
}

Pseudoaneurismo de la fibrosa del interválvulo mitroaórtico: um estúdio de caso

D’avila Suyane Belém de Lima ${ }^{1 *}$, Herbert Lima Mendes ${ }^{1}$, Acácio Vieira Machado Leite ${ }^{2}$, Carolina Naomi Shirakawa ${ }^{2}$, Lyzandra Maria de Oliveira Leite ${ }^{2}$, Lorenna Pereira Pires ${ }^{2}$, Antônio Matheus Gomes Mota ${ }^{2}$, Tarsis Zaire Ferreira da Costa², Luciano Nunes de Sousa², Iraci Alice Filizola Salmito'.

\section{RESUMO}

Objetivo: Relatar o caso clínico de uma paciente portadora de Pseudoaneurisma da Fibrosa Intervalvar MitroAórtica (PFIMA) com insuficiência mitral aguda, bem como tratamento clínico, métodos diagnósticos e tratamento definitivo empregado. Detalhamento do caso: Trata-se de um estudo de caso aprovado pelo Comitê de Ética em Pesquisa (CEP) sob o parecer 2.696.862. Paciente, sexo feminino, 18 anos, após manifestação de quadro clínico de insuficiência cardíaca aguda, foi diagnosticada como sendo de portadora de PFIMA por meio do estudo ecográfico com ecocardiograma transtorácico (ETT) e complementdo com ecocardiograma transesofágico. Havendo assim, necessidade de troca de valva mitral por bioprótese mitral que ocorreu na mesma internação sem intercorrências, tendo alta logo em seguida após ETT de controle. Considerações finais: Configura uma entidade clínica rara, de etiologia decorrente de cirurgia cardíaca ou endocardite, na maioria dos casos, ou são considerados congênitos por não terem causa definida. As complicações são a principal preocupação, visto que pode complicar com várias entidades e com isso, a morte do paciente pode ocorrer, principalmente se não for reconhecida precocemente e adequadamente tratada.

Palavras-chave: Pseudoaneurisma fibroso intervalvar mitro-aórtico, Válvula mitral, Pseudoaneurisma.

\begin{abstract}
Objective: To report the clinical case of a patient with Mitro-Aortic Intervalvar Fibrous Pseudoaneurysm (PFIMA) with acute mitral regurgitation, as well as clinical treatment, diagnostic methods and definitive treatment employed. Case details: This is a case study approved by the Research Ethics Committee (CEP) under opinion 2.696.862. An 18-year-old female patient, after presenting a clinical picture of acute heart failure, was diagnosed as having PFIMA by means of an echographic study with transthoracic echocardiography (ETT) and complemented with transesophageal echocardiography. Thus, there was a need to change the mitral valve for a mitral bioprosthesis that occurred in the same hospitalization without complications, being discharged soon after after control TTE. Final considerations: It is a rare clinical entity, of etiology resulting from cardiac surgery or endocarditis, in most cases, or are considered congenital because they have no defined cause. Complications are the main concern, since it can complicate with various entities and with this, the patient's death can occur, especially if it is not recognized early and properly treated.
\end{abstract}

Keywords: Mitro-aortic intervalvar fibrous pseudoaneurysm, Mitral valve, Pseudoaneurysm.

1 Universidade Federal do Cariri (UFCA), Juazeiro do Norte - CE. *E-mail: davila_suyane@hotmail.com

${ }^{2}$ Faculdade de Medicina Estácio de Juazeiro do Norte, Juazeiro do Norte - CE. 


\section{RESUMEN}

Objetivo: Presentar el caso clínico de un paciente con pseudoaneurisma fibroso intervalvular mitroaórtico (PFIMA) con insuficiencia mitral aguda, así como el tratamiento clínico, los métodos diagnósticos y el tratamiento definitivo empleado. Detalles del caso: Este es un estudio de caso aprobado por el Comité de Ética en Investigación (CEP) con la opinión 2.696.862. Una paciente de 18 años, tras presentar un cuadro clínico de insuficiencia cardiaca aguda, fue diagnosticada de PFIMA mediante estudio ecográfico con ecocardiografía transtorácica (ETT) y complementado con ecocardiografía transesofágica. Así, fue necesario cambiar la válvula mitral por una bioprótesis mitral que se produjo en la misma hospitalización sin complicaciones, siendo dado de alta al poco tiempo de la ETT de control. Consideraciones finales: Es una entidad clínica rara, de etiología derivada de cirugía cardíaca o endocarditis, en la mayoría de los casos, o se consideran congénitas por no tener una causa definida. Las complicaciones son la principal preocupación, ya que puede complicarse con diversas entidades y con ello, puede ocurrir la muerte del paciente, sobre todo si no se reconoce precozmente y se trata adecuadamente.

Palabras clave: Pseudoaneurisma fibroso del intervalvular mitral-aórtico, La válvula mitral, Pseudoaneurisma.

\section{INTRODUÇÃO}

A fibrosa intervalvar mitro-aórtica é uma estrutura fibrosa localizada entre a valva mitral e a valva aórtica, mais especificamente entre o folheto mitral anterior e a cúspide aórtica não coronariana. Por ser um local relativamente avascular, torna-se propenso a infecções e a trauma cirúrgico, favorecendo a formação de pseudoaneurismas (CHANDRAKUMAR $\mathrm{H}$, et al., 2020; HOSSEINSABET A e ABBASI K, 2021).

O Pseudoaneurisma da Fibrosa Intervalvar Mitro-Aórtica (PFIMA) é uma condição rara sendo definida como um pseudoaneurisma na zona interanular entre as valvas mitral e aórtica; e sua comunicação com o Trato de Saída do Ventrículo Esquerdo (TSVE), entre a válvula aórtica coronariana esquerda ou não-coronária e a cúspide anterior da valva mitral (HAN J, et al., 2016). Quanto à etiologia, a endocardite e a cirurgia valvar aórtica foram os dois fatores causais mais frequentemente associados, outras possíveis causas são o trauma torácico e a origem congênita (VARGA A, et al., 2021).

A ruptura em direção ao pericárdio produzindo tamponamento cardíaco é uma das complicações mais temidas dessa patologia. Outros eventos que podem ocorrer são: compressão de estruturas cardíacas, fístulas e embolias. O indivíduo pode apresentar no quadro clínico dor torácica, dispneia, hemoptise, insuficiência cardíaca, morte súbita e embolias sistêmicas ou cerebrovasculares (podendo resultar em isquemia). Em $12 \%$ dos casos, os pacientes apresentam-se assintomáticos (BARRANTES C e FERNANDEZ J, 2020; KIRYU K, et al., 2021).

A principal característica ecocardiográfica da mesma é a expansão na sístole e colapso na diástole. A visão paresternal do eixo longo do Ventrículo Esquerdo (VE) pode demonstrar a relação do pseudoaneurisma com o Átrio Esquerdo (AE) ou o Trato de Saída do Ventrículo Esquerdo (TSVE). A visão de eixo curto aórtico paraesternal fornece a melhor visão para avaliar a relação entre a lesão e a Átrio Direito (AD) ou aorta ascendente. A imagem de Doppler colorido é útil para detectar rupturas. O Ecocardiograma Transesofágico (ETE) é mais sensível para detectar a aorta, lesões valvares ou avaliar região subaórtica do que Ecocardiograma Transtorácico (ETT) (HAN J, et al.,2016).

Durante a sístole, a alta pressão ventricular esquerda aumenta o fluxo sanguíneo para o pseudoaneurisma e durante a diástole, o sangue flui de volta para o TSVE. O ecocardiograma mostra essa cavidade perivalvar pulsátil e, com o uso do Doppler, pode-se observar o fluxo em seu interior (CHANDRAKUMAR H, et al., 2020; ROTTA A, et al., 2018).

Atualmente, a cirurgia é o tratamento indicado mesmo em pacientes assintomáticos para evitar o desenvolvimento de complicações. A ressecção e o reparo do pseudoaneurisma podem ser realizados com ou sem a substituição da valva, podendo ser necessária uma cirurgia de revascularização (THAKRE A, et al., 2021). 
O presente relato de caso tem como objetivo representar essa condição que é raramente documentada na literatura médica mundial, através do caso clínico de uma paciente jovem portadora de PFIMA que complicou com insuficiência mitral aguda, bem como os métodos diagnósticos, o tratamento clínico e definitivo empregados.

\section{DETALHAMENTO DO CASO}

Este relato de caso respeitou os preceitos éticos sobre estudos envolvendo seres humanos, segundo a resolução de no 466/2012 do conselho Nacional de Saúde, atendendo as exigências éticas e cientificas fundamentais. Serão tomados os devidos cuidados quanto à proteção da confidencialidade do material e dados pessoais apresentados neste relato de caso, e o uso de imagens será obtido por meio da assinatura do Termo Consentimento Livre e Esclarecido.

Paciente LSM, 18 anos, sexo feminino, estudante, parda, natural e procedente de Juazeiro do Norte CE, bom grau de informação, anamnese colhida com a própria. História pregressa de internamento em setembro de 2017 em Hospital Terciário por dispneia progressiva, tosse seca e taquicardia há cerca de 20 dias, a qual evoluiu com derrame pleural bilateral e insuficiência respiratória aguda, cursando com intubação orotraqueal e assistência ventilatória mecânica.

Ao exame físico, neste internamento, foi evidenciado sopro sistólico ++++/6+ em foco mitral, realizado ETT o qual evidenciou insuficiência mitral leve a moderada, valva mitral com folhetos de espessura normal com presença de prolapso moderado sem sinais de estenose, com refluxo moderado ao estudo do doppler. Foi realizada investigação para colagenoses, todavia todos os exames foram negativos, exceto Anti-DNA: reagente (1/10). Permaneceu internada por 30 dias, recebendo alta hospitalar, com melhora do quadro e encaminhamento para serviço de cardiologia. Porém a mesma relata não ter conseguido atendimento, retornou ao mesmo hospital cerca de 15 dias depois, com quadro de astenia progressiva importante, anemia, taquicardia e tosse seca, sendo internada e inserida na central de regulação de leitos para transferência ao serviço de referência para investigação de valvopatia. Dados obtidos do relatório de alta hospitalar.

Foi admitida em 14 de dezembro de 2017 no serviço de cardiologia do Hospital do Coração Cariri, com queixa de "cansaço em repouso e inchaço nas pernas". Apresentava quadro de limitação funcional, classe funcional IV da New York Heart Association, avaliação clínico-hemodinâmica perfil quente-congesto (SOCIEDADE BRASILEIRA DE CARDIOLOGIA, 2018).

Encontrava-se hemodinamicamente estável, astênica, taquicárdica, pulsação de fúrcula esternal, a ausculta cardíaca evidenciou ritmo cardíaco regular em 2 tempos, bulhas hiperfonéticas, sopro pansistólico 4+/6+ mais audível em foco mitral com irradiação para dorso. Além de mucosas hipocoradas +/4+, edema de membros inferiores com cafico 3+/4+, abdome difusamente doloroso à palpação superficial, não foi observado refluxo hepatojugular, taquipnéica, ausculta respiratória reduzida em ambas bases sem ruídos adventícios, o Eletrocardiograma (ECG) apresentou taquicardia sinusal e sobrecarga de VE.

Como antecedentes médicos, relatou anemia moderada em 2014, tratada com medicação oral, episódios de faringoamigdalite na infância e uso de penicilina benzatina, porém negou diagnóstico prévio de febre reumática. Negou casos de autoimunidades na família.

Como rotina do serviço a paciente foi submetida ao estudo do ETT, sendo evidenciado: VE com cavidade com dimensões normais, função sistólica global normal, fração de ejeção de $72 \%$, padrão de enchimento diastólico normal; $A E$ : dimensões aumentadas em grau importante $(4,4 \mathrm{~cm})$, volume $=72 \mathrm{ml}$; $A D$ e VD: cavidade com dimensões preservada, função sistólica do VD preservada; VAo: folhetos delgados com mobilidade preservada e sem refluxo; artéria e valva pulmonares: artéria pulmonar com calibre normal, valva pulmonar normal; valva tricúspide: folhetos delgados com mobilidade preservada com refluxo moderado estimado Pressão Sistólica da Artéria Pulmonar (PSAP) em 80 milímetros de mercúrio ( $\mathrm{mmHg}$ ).

A VM demonstrava folhetos espessados com mobilidade ampla do folheto anterior que parecia "acolado" ao septo inter-atrial o plano do anel valvar se mantinha, mas havia uma redundância do folheto, conforme Figura 1, associado a refluxo importante, foi então sugerido estudo com ETE. 
Figura 1 - Ecocardiograma transtorácico.

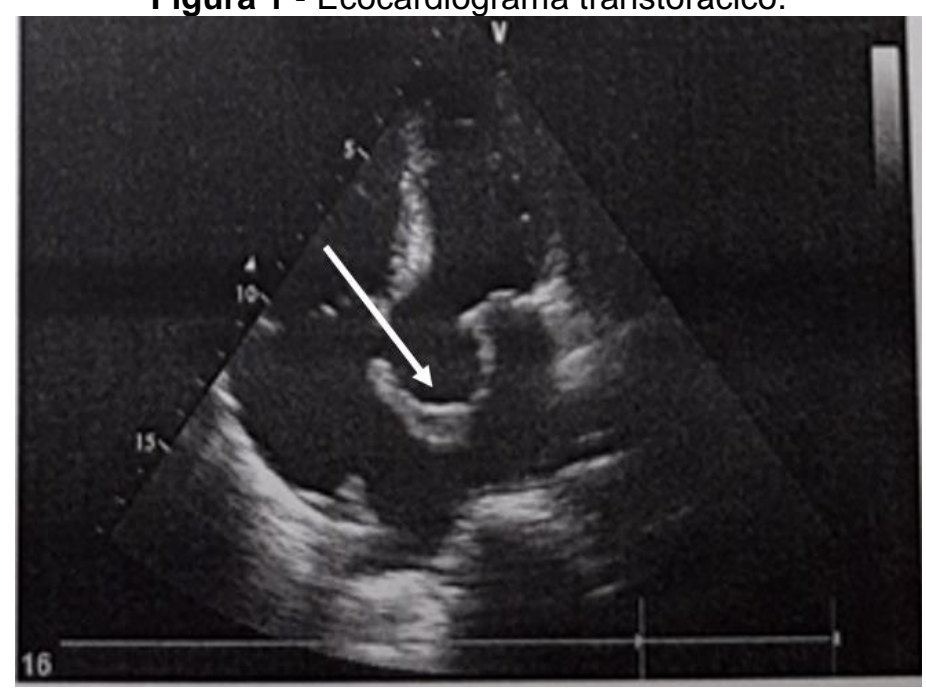

Nota: *PFIMA; Fonte: Lima DSB, et al., 2021.

Ao ETE, VM com espessamento de suas cúspides, mostrar-se boa abertura. Registrando uma maior fragilidade com dilatação aneurismática em região de fibrosa mitral-aórtica, observada na Figura 2, associado a Refluxo Mitral (RM) excêntrico direcionado para o septo interatrial de situação importante ao doppler evidenciada na Figura 3. Corroborando com os achados do ETT.

Figura 2 - Pseudoaneurisma da fibrosa intervalvar mitro-aórtica.

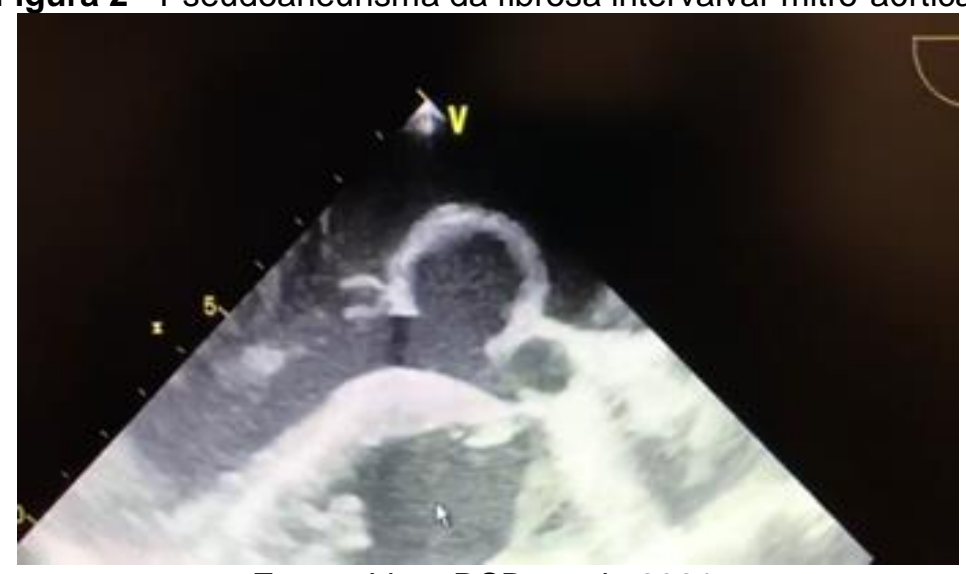

Fonte: Lima DSB, et al., 2021.

Figura 3 - Refluxo mitral.

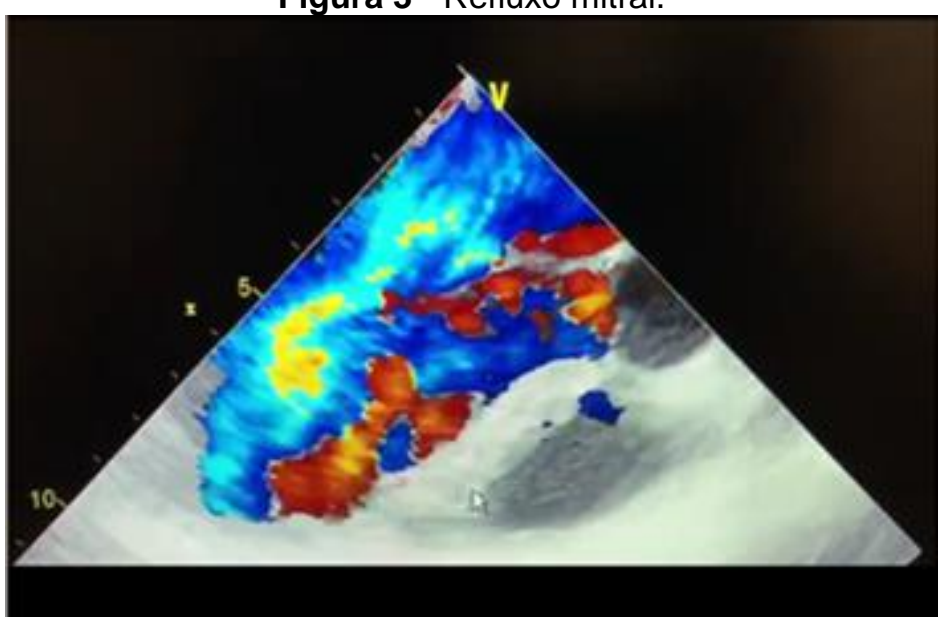

Fonte: Lima DSB, et al., 2021. 
Foi realizada cirurgia de troca de valva mitral dia 20/12/2017, com envio de valva nativa para estudo histopatológico. A esternotomia mediana longitudinal foi à via de acesso para a operação.

Sendo identificada valva mitral com refluxo importante. Folheto anterior da mitral perfurado com degeneração e abaulamento importante como aneurisma, nota-se ainda degeneração também da estrutura fibrosa do anel e de parte do folheto posterior que pode ser vista na Figura 4. Realizado troca por bioprótese mitral $\mathrm{n} 29$.

Paciente evoluiu bem no pós-operatório (PO), realizando fisioterapia respiratória, sendo dada alta no $5^{\circ}$ PO para enfermaria cirúrgica, em respiração espontânea ao ar ambiente sem sinais de desconforto e redução gradativa do edema de membros inferiores, ausculta cardíaca com ritmo regular e sem sopros.

Figura 4 - Pseudoaneurisma da fibrosa intervalvar mitro-aórtica.

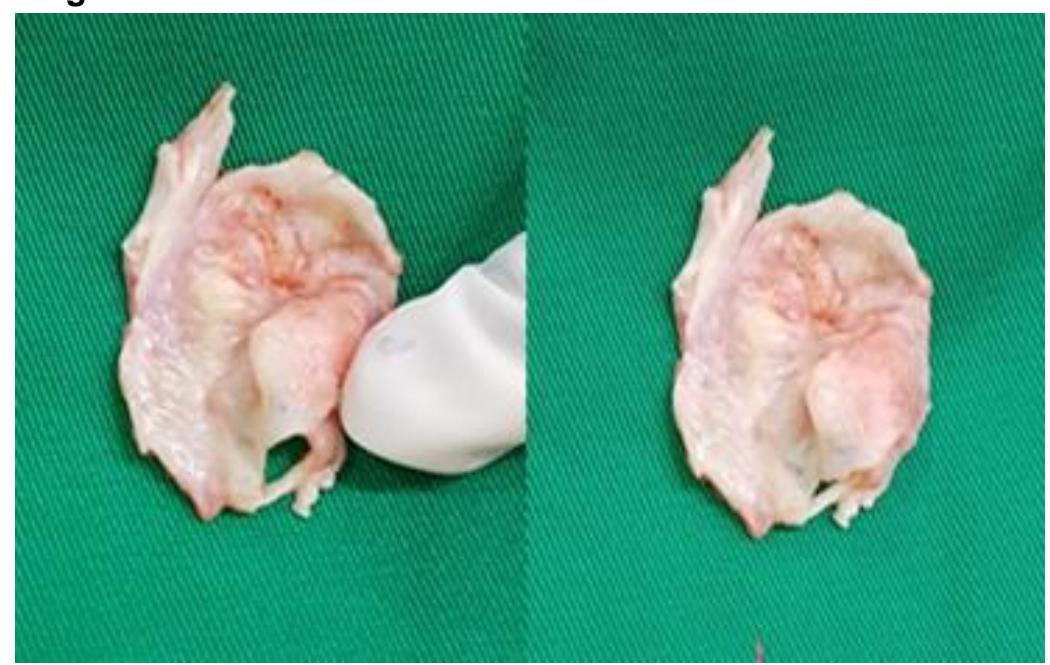

Fonte: Lima DSB, et al., 2021.

O ETT de controle realizado dia 26/12/2017 sendo evidenciada prótese biológica com boa função, área estimada em $2,1 \mathrm{~cm}^{2}$ e gradientes máximo e mínimo de 8 e $4 \mathrm{mmHg}$ respectivamente, sem refluxo; valva tricúspide com refluxo leve estimado PSAP em $45 \mathrm{mmHg}$.

O estudo histopatológico revelou achados inespecíficos, tecido conjuntivo denso espessado com processo inflamatório crônico e material fibrinóide.

Foi dada alta a paciente dia 29/12/2017 em bom estado geral com prescrição de furosemida 40mg 2 vezes ao dia, aldactone $25 \mathrm{mg} 1 \mathrm{vez}$ ao dia, neutrofer $300 \mathrm{mg}$, com retorno marcado para 30 dias. Na consulta de retorno a paciente apresentou-se em excelente aspecto geral, com relato de ganho de peso, sem edemas, sem dispneia, sendo reduzida furosemida para uma vez ao dia. Orientado acompanhamento ambulatorial na clínica escola da Estácio FMJ.

\section{DISCUSSÃO}

PFIMA é uma rara condição cuja etiologia permanece uma questão de debate. O PFIMA pode se formar após um infarto agudo do miocárdio, por trauma torácico, após manipulação da área durante cirurgia cardíaca, por endocardite infecciosa ou pode ser congênito (BARRANTES C e FERNANDEZ J, 2020). Entretanto, a maioria dos pacientes não têm etiologias claras e foram considerados de origem "congênitos" (HARRINGTON JK, et al., 2017). Um aspecto do caso aqui relatado contempla a hipótese acerca dessa possível etiologia congênita, dada à idade jovem da paciente e a inexistência de infecção prévia.

A fibrose mitro-aórtica é uma área da via de saída do ventrículo esquerdo. Esta estrutura está em justaposição com uma cunha epicárdica de gordura que fica entre a parede posterior da aorta e 0 átrio esquerdo (AE). O PFIMA se projeta externamente entre a raiz aórtica e o AE. A perfuração pode resultar em 
comunicação fistulosa com qualquer uma das câmaras e embora as principais causas etiológicas de PFIMA sejam infecções e traumas cirúrgicos, o jato de regurgitação aórtica também contribui para sua formação (AGUS HZ, et al., 2018; BAGH I, et al., 2021; PRAVEEN CH, et al., 2021).

As características clínicas comumente relatadas nestes pacientes incluem falta de ar, sinais e sintomas de insuficiência cardíaca, infecção ativa (de endocardite infecciosa), dor no peito, angina atípica. Porém, alguns permanecem assintomáticos onde o pseudoaneurisma é diagnosticado incidentalmente (CHANDRAKUMAR $\mathrm{H}$, et al., 2020). A paciente do caso relatado apresentou-se sintomática, assim como é mais comumente relatada quando na presença da PFIMA.

Um PFIMA pode causar condições de risco de vida, como ruptura no átrio esquerdo levando à comunicação do trato de saída do ventrículo esquerdo para o átrio esquerdo. No entanto, um pseudoaneurisma pode permanecer relativamente estável por muito tempo, apresentando-se como uma cavidade pulsátil com expansão sistólica. Se crescer, pode comprimir a artéria pulmonar ou a artéria coronária esquerda, causando angina. A embolização de um trombo formado em um PFIMA pode causar acidente vascular cerebral (MURETTI M, et al., 2019). A PFIMA é uma condição patológica rara que requer uma base estatística sólida para conclusões diversas, como etiologia, tratamento e conduta no geral.

A formação de fístulas entre as cavidades cardíacas constitui outra forma de complicação. Uma fístula pode se desenvolver entre o VSVE e o AE ou a aorta, um fluxo excêntrico pode ser visualizado imitando um jato mitral regurgitante. Assim, qualquer nova regurgitação mitral excêntrica detectada após a substituição prévia ou recente da valva aórtica deve levantar suspeita de formação fistulosa (ŞAHAN E, et al., 2015). A evolução dessa complicação está associada a um elevado índice de morbidade, podendo ocorrer mesmo após anos de um reparo cirúrgico eficaz. Deve-se, portanto, ser feito um acompanhamento contínuo desses pacientes, para que na vigência de uma fístula seja feita correção imediata (ABDALLAH H, et al., 2017). No caso índice, foi observado na análise macroscópica da valva mitral, uma lesão perfurada a nível de folheto anterior, que intensificou o RM e levou a descompensação clínica da paciente.

O diagnóstico desta entidade pode ser feito por diferentes modalidades de imagem, como ETT, ETE, tomografia computadorizada e ressonância magnética. O ETE é preferível, visto que possui uma maior sensibilidade quando comparado ao ETT. O aspecto ecocardiográfico do PFIMA é o de uma falsa luz abaixo do anel atrioventricular. A cavidade pseudo-aneurismática exibe marcada pulsatilidade com expansão sistólica e colapso diastólico e se comunica com a TSVE. Ao doppler colorido demonstra fluxo para dentro da cavidade na sístole e fluxo reverso na diástole e pode ajudar a representar uma ruptura do pseudoaneurisma nas estruturas adjacentes mais frequentemente o AE, a aorta e o pericárdio (APOSTOLIDOU E, et al., 2017). O ETT também fornece valor na avaliação da gravidade das lesões valvares e da função ventricular (NUNES M e FERRARI TCA 2020).

O pseudoaneurisma é uma manifestação de infecção local descontrolada e uma indicação para gerenciamento cirúrgico de emergência (Classe I, Nível de evidência B) (ROTTA A, et al., 2018). Casos complicados, sintomáticos como o apresentado neste relato, com características de alto risco devem ser excisados cirurgicamente. O procedimento cirúrgico é geralmente complexo e pode requerer substituição de valva, reparo de sua raiz e reconstrução do PFIMA. Seu curso natural não complicado é largamente desconhecido, uma vez que, no momento do diagnóstico, a maioria dos pacientes é submetida à cirurgia para tratar sintomas ou prevenir complicações. Nossa paciente foi admitida em franca insuficiência cardíaca, refluxo importante ao exame físico e risco iminente de óbito caso não fosse realizado tratamento invasivo.

O conhecimento do perfil clínico e história natural do PFIMA é limitado. Outras complicações que podem surgir são formação de fístula, compressão do coronário pulmonar ou artéria adjacente (principal esquerda, descendente anterior esquerdo ou circunflexo), trombose (com tromboembolismo), infecção ou ruptura com tamponamento de pericárdio e morte súbita (SPAMPINATO RA, et al., 2013). No entanto, houve relatos em estudos, sem intercorrências até 5 anos após o diagnóstico, no seguimento de PFIMA, porém se tratavam de PFIMA sem complicações. Geralmente, uma abordagem de espera vigilante com imagem seriada e encaminhamento para cirurgia, caso ocorram complicações ou rápida expansão, pode ser válida para pacientes assintomáticos e que apresentam alto risco operatório (GIN A, et al., 2011). 
O PFIMA é uma condição rara com exíguos relatos na literatura médica mundial, que se não reconhecido precocemente e tratado adequadamente pode acarretar desfecho potencialmente fatal. A etiologia é desconhecida na maioria dos casos, o que dificulta a identificação dos portadores e o acompanhamento dos pacientes com fatores de risco, tendo em vista tal dificuldade, a identificação acontece, na maioria das vezes, em fase avançada da doença, aumentando a necessidade de recursos mais invasivos para resolução. Fazse necessário, portanto, um acesso adequado ao serviço especializado para um desenlace satisfatório, como realizado no caso supracitado.

\section{REFERÊNCIAS}

1. ABDALLAH H, et al. Fistula formation following repair of a pseudoaneurysm of the mitral-aortic intervalvular fibrosa A rare complication. Journal of Cardiology Cases, 2017; 15(5): 170-172.

2. AGUS HZ, et al. Infective endocarditis of a bicuspid aortic valve complicated by septal aneurysm and mitral-aortic intervalvular fibrosa pseudoaneurysm. Turk Kardiyol Dern Ars, 2018; 46(2): 147-150.

3. APOSTOLIDOU E, et al. Pseudoaneurysm of the Mitral-Aortic Intervalvular Fibrosa: A Case Series with Literature Review. Cardiovascular Imaging Case Reports, 2017; 1(6): 221-226.

4. BAGH I, et al. Pseudoaneurysm of the mitral-aortic intervalvular fibrosa with fistulous communication to the left atrium causing congestive heart failure. Clinical Case Reports, 2021; 9(6): 9:04301.

5. BARRANTES C, FERNANDEZ J. Pseudoaneurisma de la fibrosa mitroaórtica: un hallazgo de una complicación tardía. Horizonte Médico (Lima), 2020; 20(4): 917.

6. CHANDRAKUMAR $\mathrm{H}$, et al. 'Pseudoaneurysm of the Mitral-Aortic Fibrosa in the Absence of Valvulitis.' American Journal of Medical Case Reports, 2020; 8(12): 478-480.

7. GIN A, et al. Pseudoaneurysms of the mitral-aortic intervalvular fibrosa: survival without reoperation. American Heart Journal, 2011; 161(1): 130-131.

8. HAN J, et al. Echocardiographic Diagnosis and Outcome of Pseudoaneurysm of the Mitral-Aortic Intervalvular Fibrosa: Results of a Single-Center Experience in Beijing. Medicine, 2016; 95(11).

9. HARRINGTON JK, et al. Congenital pseudoaneurysm of the mitral-aortic intervalvular fibrosa: a case report. Cardiology in The Young, 2017; 27(8): 1647-1650.

10. HOSSEINSABET A, ABBASI K. Pseudoaneurysm of the mitral-aortic intervalvular fibrosa in a patient with a history of bentall operation. Ann Card Anaesth, 2021; 24: 120-1.

11. KIRIU K, et al. Tratamento cirúrgico para pseudoaneurisma descoberto acidentalmente da fibrosa intervalvular mitralaórtica com válvula aórtica bicúspide, aneurisma da aorta ascendente e isquemia miocárdica: um relato de caso. Cardiothorac Surg 29, 2021; 12.

12. MURETTI M, et al. Surgical Repair of Mitral - Aortic Intervalvular Fibrosa Pseudoaneurysm. Texas Heart Institute, Texas Heart Institute Journal, 2019; 46(2): 147-150.

13. NUNES MCP, FERRARI TCA. Mitral-Aortic Intervalvular Fibrosa: A Hidden Region Associated With Infective Endocarditis Complications. JACC Case Rep, 2020; 2(8): 1217-1219.

14. PRAVEEN CH, et al. Pseudoaneurysm of Mitral-Aortic Intervalvular Fibrosa - A Case Report. MAMC Journal of Medical Sciences, 2021; 7(2): 168.

15. ROTTA A, et al. Pseudoaneurisma de la fibrosa mitro aórtica en endocarditis de aorta bicúspide: A propósito de un caso. Revista Medica Herediana,2018; 29(3): 168-172.

16. SAHAN E, et al. Pseudoaneurysm of the mitral-aortic intervalvular fibrosa. Herz, 2015; 40(2): $182-189$.

17. SOCIEDADE BRASILEIRA DE CARDIOLOGIA. Comitê Coordenador da Diretriz de Insuficiência Cardíaca. Diretriz Brasileira de Insuficiência Cardíaca Crônica e Aguda. Arquivo Brasileiro de Cardiologia, 2018; 111(3): 436-539

18. SPAMPINATO RA, et al. Pseudoaneurysm of the mitral-aortic intervalvular fibrosa as a complication after minimally invasive mitral valve repair. Interactive Cardiovascular and Thoracic Surgery, 2013; 16(3): 396-398.

19. THAKRE A, et al. Um Caso Interessante de Pseudoaneurisma da Fibrosa Intervalvar Mitral-Aórtica. J Indian Acad Echocardiogr Cardiovasc Imaging, 2021; 5: 40-2.

20. VARGA A, et al. Native Aortic Valve Endocarditis Complicated by Splenic Infarction and Giant Mitral-Aortic Intervalvular Fibrosa Pseudoaneurysm - A Case Report and Brief Review of the Literature. Diagnostics, 2021; 11: 251. 